\title{
Effects of employer management on employee recruitment, satisfaction, engagement, and retention on large US dairy farms
}

\author{
Stanley J. Moore, ${ }^{1 *} \odot$ Phillip T. Durst, ${ }^{1} \oplus$ Caroline Ritter, ${ }^{2} \odot{ }^{\circ}$ Diego Nobrega, ${ }^{2} \odot$ and Herman W. Barkema ${ }^{2} \odot$ \\ ${ }^{1}$ Michigan State University Extension, Michigan State University, East Lansing 48824 \\ ${ }^{2}$ Department of Production Animal Health, Faculty of Veterinary Medicine, University of Calgary, 2500 University Dr., Calgary, AB, T2N 1N4, \\ Canada
}

\section{ABSTRACT}

Lack of employee engagement and satisfaction and high turnover rate of employees are major problems on some US dairy farms. Farm adoption of human resource management (HRM) practices varies widely. Using feedback from 168 employees from 12 large dairy farms, our objectives were to understand employee perceptions of HRM strengths and weaknesses and their influence on (1) employee satisfaction, (2) retention of employees, (3) willingness of employees to recommend the farm as a place to work, and (4) level of employee engagement on the farm. Employees who rated their employers higher for almost any management-related question (MRQ) were more satisfied in their jobs and more likely to recommend their place of work to other potential employees. Employees reported a higher intention to stay on the farm when employers scored higher on MRQs connected to employer-employee relationships. Employees who rated "Relationships" (a component consisting of 5 MRQs) higher were more likely to have a higher rate of satisfaction, were more likely to intend to stay at their job, and were more likely to recommend their place of work to others. No single management question was positively related to employee engagement (as measured by employees having and sharing ideas to improve the business); however, ethnicity, gender, job role, duration of employment, and employee's selfappraisal of their desire to learn and commitment to the farm were each associated with engagement. Female employees were less likely to provide ideas to their employers (compared with male employees), as were Spanish-speaking employees (compared with Englishspeaking employees). Differences between Spanish- and English-speaking employees were also present in measures such as intention to stay (shorter) and willingness to recommend the farm as a place to work (higher). Employees who rated themselves higher on their desire

Received December 8, 2019.

Accepted April 25, 2020.

*Corresponding author: moorest@msu.edu to learn and commitment to the farm were more likely to provide ideas to their employers, as were longer-term employees. In conclusion, we inferred that dairy farm management can improve employee retention and engagement through improved use of HRM practices.

Key words: employee management, dairy employees, farm personnel

\section{INTRODUCTION}

Worldwide, the size of dairy farms and the number of employees continue to increase (Barkema et al., 2015). In the top 17 US dairy states from 2009 to 2014, medium (100-499 cows) and large (500+ cows) operations had 12 and $24 \%$ increases in herd size, respectively, with intentions to continue to grow by $26 \%$ over the next 5 yr (all farm sizes; USDA-NAHMS, 2016). Success in farm expansion plans will hinge largely on availability of a reliable, productive labor force and the farm's ability to recruit and retain employees who are committed to the farm's success. Dairy farm managers who adopt and continue to improve upon well-recognized human resource management (HRM) principles are likely to have better employee satisfaction, retention, and recruitment success (Durst et al., 2018).

Many of today's dairy farms in the United States have transitioned from predominantly family labor to being fully dependent on nonfamily labor, including a now-majority immigrant labor force milking cows on larger farms (Adcock et al., 2015). This lack of longterm experience with nonfamily labor leaves farm managers with a smaller toolbox of HRM skills or, at a minimum, a toolbox that has not been used to its full potential. Adoption of management practices is affected by a complex array of factors, including problem awareness and perception of responsibility, effectiveness of recommended strategies, farmers' (perceived) ability to implement recommended management practices, and perceived benefits and disadvantages (Ritter et al., 2017).

To meet management's objectives of a productive, satisfied labor force with a low turnover rate, and em- 
ployees who recommend job openings to others and who are engaged, managers will need to meet the basic sociological needs of their employees. Employees, like all people, have a need for autonomy, relatedness, and competence (Ilardi et al., 1993; Van den Broeck et al., 2010). According to Fowler (2014), "Autonomy can be defined as the need to perceive we have choices and control of our actions; Relatedness is the need to care about and be cared about and contribute to something greater than ourselves; Competence is the need to feel effective at doing a job and expanding your skills."

Management has a responsibility to create a farm culture that recognizes these needs and creates opportunities for employees to grow in all 3 of the areas of autonomy, relatedness and competence. Some HRM research has been done on dairy farms (Stup et al., 2006; Erskine et al., 2015; Durst et al., 2018); however, application of these principles has not been systematic because, historically, smaller farms used primarily family employees and labor availability was greater (Adcock et al., 2015). In addition, most HRM research has been done in businesses that have a different employee base than agriculture. For instance, in the study by Van den Broeck et al. (2010), 95 (8\%) of the 1,185 employees surveyed considered themselves to be blue collar workers (nonagricultural), whereas the remaining 1,090 (92\%) considered themselves to be administrative personnel, professionals, or managers. Although this research is valuable to the overall human resources field of study, it cannot automatically be generalized to agriculture, which often involves an immigrant workforce. The combined lack of training, training materials, and application reduces adoption of HRM practices on farms, with a potential detrimental impact on employee recruitment and retention.

Durst et al. (2018) identified 6 common management areas as weaknesses on many dairy farms: failure to specify goals, failure to encourage employee input, employee-to-employee problems, lack of communication, failure to provide training, and failure to provide specific positive feedback. These align well with the basic psychological needs cited by Ryan and Deci (2000): autonomy, relatedness, and competence. Although these 3 needs are not completely independent, as pointed out by Fowler (2014), the combination of these needs affects employee attitudes toward their work and desire to stay.

If good HRM practices on farm influence employee satisfaction, willingness to recommend, desire to stay, and employee engagement, employees' perception of HRM can be measured and impacts on employees determined. Our objectives were, therefore, to understand employee perceptions of HRM strengths and weaknesses and their influence on (1) employee satisfaction,
(2) employee retention, (3) willingness of employees to recommend the farm as a place to work, and (4) level of employee engagement on the farm.

\section{MATERIALS AND METHODS}

\section{Recruitment of Farms}

Farms were invited to participate in this project through a variety of avenues, including distribution of printed materials, verbal communications, and personal approaches. Dairy farm owners voluntarily participated in the project and allowed their employees to voluntarily participate in this project, creating a convenience sampling. Thirteen farms voluntarily enrolled in the program between November 2012 and May 2014, with the majority from Michigan $(\mathrm{n}=7)$, and the remainder from New York $(\mathrm{n}=2)$, Pennsylvania $(\mathrm{n}=2)$, and Connecticut $(\mathrm{n}=2)$. Enrollment was limited to farms with $\geq 10$ employees to ensure anonymity of employees and to provide sufficient feedback from employees on each farm to owners and managers. No distinction was made between full-time and part-time employees. The enrollment process included a personal visit by project personnel to each farm, followed by emails and phone conversations. Each farm paid an enrollment fee based on the number of individuals employed on their farm. This enrollment fee covered the cost of the bilingual interviewer recruited for this project.

\section{Questionnaires}

The employee questionnaire (see Supplemental File S1; https://doi.org/10.3168/jds.2019-18025) included 21 ordinal scale questions ( $\mathbf{Q}$; scale of 1 to 5 , with descriptors) and 8 open-ended questions. Ordinal scale questions were designed with the most positive response indicated by a 5 (with the exception of Q28, for which 4 answers were possible); descriptors were used for high and low ratings in all cases and for each rating in some cases. On all scale questions, employees were also invited to provide supporting comments to their answers.

Some questions (e.g., Q5, 6, 25, 26, 28) were about employees' work attitudes, some were about their work environment (e.g., Q13-19), and others were about their perception of their supervisors' attitudes and behavior (Q20, 21). Thus, the questionnaire attempted to capture various aspects that affected evaluation of the work environment, whether directly or indirectly affected by management, for employees.

A questionnaire was also prepared for employers (owners and managers). The employer questionnaire (Supplemental File S2; https://doi.org/10.3168/ 
jds.2019-18025) included 21 ordinal scale questions and 5 open-ended questions that asked to rate how they thought their employees would respond to each management-related question (MRQ) of the employee questionnaire. Managers who were not owners were asked to complete the survey because of their role in supervising employees even though they too were employees. They were told that they could also complete the employee survey if they desired. Because of anonymity of the employee surveys, it is unknown whether any of the managers did.

Study design and questionnaires were reviewed and approved by the Michigan State University Institutional Review Board and were given exempt status (IRB\# $\mathrm{x} 12-799 \mathrm{e}$ ), as well as being reviewed internally by 4 Michigan State University faculty members and externally by 2 dairy producers. Pilot testing and validation of the survey was not completed.

Employee responses to MRQs provided insight into their perceptions of key areas (components) of management: Relationships (Q8, 18, 20, 23, 24); Feedback and Fairness (Q10, 16, 21, 22); Goals and Direction (Q11, 12, 13, 17); and Self-Appraisal (Q25, 26).

\section{Data Collection}

Project personnel met with employers to review the project during the enrollment visit. Employers were asked to fill out the employer questionnaire and to provide additional data, including number of employees, number of replacement hires in the past 12 mo, number of cows at the beginning and end of the previous year, total employee payroll, and total amount of milk shipped. As the project proceeded, data were collected for more than 1 yr. Employers were also provided with paper copies of the employee questionnaire in English and in Spanish. Employers were asked to distribute these employee questionnaires to their employees in preparation for employee phone interviews. Employees were asked not to fill out the paper questionnaire but instead to familiarize themselves with what would be asked in the phone interview before calling the interviewer.

Employee phone interviews were voluntary and conducted by one native Spanish-speaking interviewer who was also fluent in English and Portuguese. Employees from each farm were given a block of days and times in which they could initiate a call to the interviewer to conduct the interview. All callers remained anonymous and were identified by the interviewer by assigned number, name of farm, and preferred language (English- and Spanish-speaking employees; 5 Portuguese-speaking respondents were included with the Spanish-speaking group for analysis). Over $75 \%$ (ranging from 55 to
$100 \%$ within farms) of employees on the project farms called in to be interviewed. Employee answers provided in Spanish or Portuguese were translated into English after the interview. Translation was performed by the native Spanish-speaking interviewer, who was also fluent in English and Portuguese.

\section{Statistical Analyses}

Statistical analyses were performed using Stata SE 12 (StataCorp LP, College Station, TX), and $P<0.05$ was considered significant. Employees' perception of management areas is reported for each MRQ as either a low rating (1-3 on an ordinal scale of $1-5)$ or a high rating (4-5 on the same scale). Median MRQ employee response was a 4 or 5 ; hence, to have sufficient numbers of participants in each category, a response of 3 was included in the low category. In addition, we considered it important to compare "above average" (or high) with lower ratings. Employees' answers to 4 questions on the survey were considered as outcome variables in a separate analysis: (1) How satisfied are you working at this dairy? ("satisfaction"; Q5; scale 1-5); (2) How much longer do you want to work here? ("intend to stay"; Q28; scale 1-4); (3) Would you recommend this workplace to others? ("recommend to others"; Q7; scale 1-5); and (4) How often do you come up with ideas to improve the operation? ("frequency of ideas"; Q27; scale 1-3), transformed from an open-ended question. Responses to the open-ended question "How frequently do you have ideas to improve the business, whether you share them or not?" (Q27) were divided into 3 categories: (1) frequently have ideas to improve the business (defined as when respondent provided a frequency of at least once a month or a response that indicated very frequently); (2) occasionally (defined as when respondent indicated a frequency of less than once per month or a response that indicated occasionally); and (3) never (responses such as "I just do what they tell me to do" and "have ideas but do not share them"). First, the association of each individual MRQ with the 4 outcome measures was assessed. Second, a principal components analysis (PCA) was used to create summary measures (i.e., principal components) of individual variables. Third, univariable associations between the 4 outcome variables and all explanatory variables, including the 4 extracted components from the PCA analysis, were assessed. Finally, explanatory variables with $P<0.20$ in univariable analysis were submitted to multivariable ordinal mixed-effects regression analyses to assess their relationship to the 4 aforementioned outcome variables.

Nonparametric Tests. First, responses to the 4 outcome variables were compared with individual MRQs using the Mann-Whitney U test. In that regard, 
satisfaction ratings were compared among employees who gave ratings of either $1-3$ or $4-5$ to various MRQ. Similarly, we compared employees' intention to stay, their willingness to recommend the farm as a place to work, and their frequency of ideas depending on their ratings of MRQ.

$\boldsymbol{P C A}$. A PCA was conducted to extract composite components from sets of MRQ containing repeated information. Four analyses were done, each using a different set of questions categorized in 4 components: Relationships, Feedback and Fairness, Goals and Direction, and Self-Appraisal. These 4 deductively designed composite components were created based on existing management literature and presence of at least one suitable component following the PCA that was indicative of validity or communality of MRQ (Hoyle, 2012; Brown, 2014). Suitability of components was evaluated based on their eigenvalues $>1$ and Cronbach's $\alpha>0.55$ (Fabrigar and Wegener, 2011; Tavakol and Dennick, 2011; Babbie, 2015). For further analysis, only the first component derived from the PCA was retained and evaluated. Eigenvalues for components ranged from 1.26 to 2.59, whereas Cronbach's $\alpha$ for components ranged from 0.40 to 0.74 .

Component 1 (Relationships) described the relationship with coworkers and between employees and employers and included the following items: Rate the teamwork within the dairy (Q8), Talk with supervisor about problems? (Q18), Rate employer: working to improve the operation (Q20), Open and honest communication with supervisor? (Q23), and Rate relationship with supervisor (Q24).

Component 2 (Feedback and Fairness) described feedback from employers and perceived fairness toward employees and included the following items: Are rules applied fairly and consistently? (Q10), Frequency of feedback, positive or negative? (Q16), Rate employer: holding employees accountable (Q21), and Frequency of recognition/praise in last $15 \mathrm{~d}$ (Q22).

Component 3 (Goals and Direction) represented employees' perceptions regarding their knowledge of and contributions to farm goals and their role on the farm and included the following items: How well are farm goals communicated to you? (Q11), Do you know how your work contributes to the goals? (Q12), Do you know what is expected from you? (Q13), and Is it clear to you who your supervisor it? (Q17).

Component 4 (Self-Appraisal) represented employees' self-evaluation in regard to their interest in learning and commitment to the farm success and included the following items: Employees rated self: interest in learning (Q25) and employee rated self: commitment to farm success (Q26). Although this component had a Cronbach's $\alpha$ of 0.40 , we believed that this component represented a theoretically relevant concept. Therefore, and because the component included only 2 variables that suppressed that $\alpha$ value, we decided to retain this component and submit it to regression analysis.

Regression Modeling. Associations of explanatory variables, including the 4 extracted components from the PCA analysis, with the 4 outcome variables, were assessed using univariable ordinal regression analysis. Here, the category on either end of the ordinal scale that had the most employee replies was selected as the baseline. Because several employees per farm were interviewed, multilevel regression analysis was considered for employee-specific variables, with "farm" being assessed as a random effect. However, if the likelihood ratio test comparing the multilevel model with the model that did not account for clustering by farm was not statistically significant, we proceeded with the simpler model, assuming independence of observations from same farms. Then, we evaluated whether the model met the proportional odds assumption (Stata command "omodel" for non-multilevel models and "gllamm" for multilevel models). When models did not meet the proportional odds assumption, logistic regression models were used to report odds ratios for each "cut" of outcome categories (e.g., after collapsing cells with low counts, the outcome "satisfaction" had 4 levels with 3 cuts, whereas the outcome "Recommend to others" only had 3 levels, with 2 cuts between them).

Finally, all variables with $P<0.20$ in the univariable analysis were considered for the multivariable mixed-effects logistic regression analyses. To improve interpretability and model convergence, outcome variables were collapsed to be binary. If there was multicollinearity among explanatory variables, variables were excluded based on correlation with other variables. Testing for clustering within farm was done as described for the univariable analysis. Two-way interaction terms were included in the multivariable model for demographic variables at the employee level if both terms were $P$ $<0.20$. To identify the most important predictors for employees' satisfaction, intention to stay, engagement, and their willingness to recommend the farm as a workplace, manual backward elimination was used on all 4 logistic regression models. Presence of effect modification was assessed by evaluating Wald test $P$ values of interaction terms. After removal of a variable, the remaining predictors were evaluated for confounding (i.e., $30 \%$ change in the estimate) by the removed variable (this was not done for interaction terms). If there was confounding, the variable remained in the model. The likelihood ratio test was applied after every variable removal to compare the full model with the reduced model. If the test was significant, the last 
removed variable was inserted back into the model and backward elimination was terminated.

\section{RESULTS}

In total, 174 employees of 13 farms participated in the employee questionnaire (see Supplemental File S1; https://doi.org/10.3168/jds.2019-18025). One farm was removed from the study because only 6 of 27 employees answered the questionnaire, failing to reach the minimum of $50 \%$ of employees responding. Results from the remaining 168 employees from 12 farms are reported from Michigan (61\%), New York (24\%), Pennsylvania (11\%), and Connecticut (4\%). Tables 1, 2, 3, and 4 show employee responses to MRQs; Tables 5, 6, and 7 present results of univariable and mutivariable regression analyses.

\section{Employee Satisfaction}

For Q5: How satisfied are you in working at this dairy? mean and median responses were 4.1 and 4.0, respectively, on an ordinal scale of $1-5$, where $1=$ not at all satisfied and $5=$ most satisfied.

Employees who rated their employer higher on MRQ also reported higher satisfaction compared with employees who rated their employer lower on these questions (16 out of 18 MRQs; Table 1), except for "frequency of feedback" (Q16) and "employee rate self: interest in learning" (Q25). Employee satisfaction was most positively associated with employees' relationship with, and opinions about, their supervisor; for example, "relationship with supervisor" (Q24; 3.0 vs. 4.3, low MRQ vs. high MRQ, respectively), "rate employer: working to improve the operation" (Q20; 3.2 vs. 4.4), "talk with supervisor about problems" (Q18; 3.1 vs. 4.2), and "open and honest communication with supervisor" (Q23; 3.3 vs 4.4; Table 1). Additionally, responses to the questions "know what's expected of you" (Q13) and "rules applied fairly and consistently" (Q10) were positively associated with employee satisfaction (3.1 vs. 4.2 and 3.3 vs. 4.4 , respectively).

In univariable analysis, employees who perceived having sufficient tools and equipment (Q14), more frequent training (Q15), or who felt they had more independence to do their jobs (Q19) were more satisfied (Table 5). Furthermore, all PCA components were positively associated with employees' satisfaction (Table 6): employees who rated the Relationships component with coworkers and supervisors higher were more satisfied with their workplace, as were employees who gave higher ratings to the Feedback and Fairness, Goals and Directions, and Self-Appraisal components. After collapsing variables in the multivariable regression analysis, the Relationships and Goals and Directions components remained significantly associated with employee satisfaction (Table 7 ).

\section{Retention}

For Q28: If it were up to you, how much longer would you remain in a position on this farm? mean and

Table 1. Employees' satisfaction in relation to their perception of management strengths and weaknesses

\begin{tabular}{|c|c|c|c|c|}
\hline & & \multicolumn{2}{|c|}{ Average satisfaction $^{1}[$ mean $(\mathrm{n})]$} & \multirow[b]{2}{*}{$P$-value } \\
\hline \multicolumn{2}{|c|}{ Management-related question (MRQ) } & MRQ low rating ${ }^{2}$ & MRQ high rating ${ }^{3}$ & \\
\hline 8. & Rate the teamwork within dairy & $3.7(61)$ & $4.3(104)$ & $<0.001$ \\
\hline 10. & Rules applied fairly and consistently? & $3.3(43)$ & $4.4(125)$ & $<0.001$ \\
\hline 11. & How well are farm goals communicated to you? & $3.8(60)$ & $4.3(107)$ & $<0.01$ \\
\hline 12. & Do you know how your work contributes to the goals? & $3.2(21)$ & $4.2(145)$ & $<0.001$ \\
\hline 13. & Know what is expected from you? & $3.1(16)$ & $4.2(152)$ & $<0.001$ \\
\hline 14. & Have tools and equipment needed to do job right? & $3.7(60)$ & $4.3(107)$ & $<0.001$ \\
\hline 15. & Frequency of training to improve skills? & $4.0(110)$ & $4.3(50)$ & 0.04 \\
\hline 16. & Frequency of feedback, positive or negative? & $4.0(73)$ & $4.1(93)$ & 0.54 \\
\hline 17. & Is it clear to you who your supervisor is? & $3.2(23)$ & $4.2(144)$ & $<0.001$ \\
\hline 18. & Talk with supervisor about problems? & $3.1(25)$ & $4.2(143)$ & $<0.001$ \\
\hline 19. & Given independence to do your job? & $3.3(24)$ & $4.2(143)$ & $<0.001$ \\
\hline 20. & Rate employer: working to improve operation & $3.2(44)$ & $4.4(122)$ & $<0.001$ \\
\hline 21. & Rate employer: holding employees accountable & $3.6(53)$ & $4.3(114)$ & $<0.001$ \\
\hline 22. & Frequency of recognition/praise in last $15 \mathrm{~d} ?$ & $3.8(96)$ & $4.4(72)$ & $<0.01$ \\
\hline 23. & Open and honest communication with supervisor? & $3.3(52)$ & $4.4(109)$ & $<0.001$ \\
\hline 24. & Rate relationship with supervisor & $3.0(28)$ & $4.3(139)$ & $<0.001$ \\
\hline 25. & Employee rate self: interest in learning & $3.8(10)$ & $4.1(158)$ & 0.38 \\
\hline 26. & Employee rate self: commitment to farm success & $3.6(22)$ & $4.2(145)$ & $<0.01$ \\
\hline
\end{tabular}

${ }^{1}$ Mean employees' satisfaction in working at this dairy on a scale from 1 to 5 , where $1=$ not at all satisfied and $5=$ most satisfied.

${ }^{2} \mathrm{MRQ}$ low rating: employees rated the MRQ at $1-3$.

${ }^{3} \mathrm{MRQ}$ high rating: employees rated the MRQ at $4-5$. 
Table 2. Employees' intention to remain on the job as related to their perception of management strengths and weaknesses

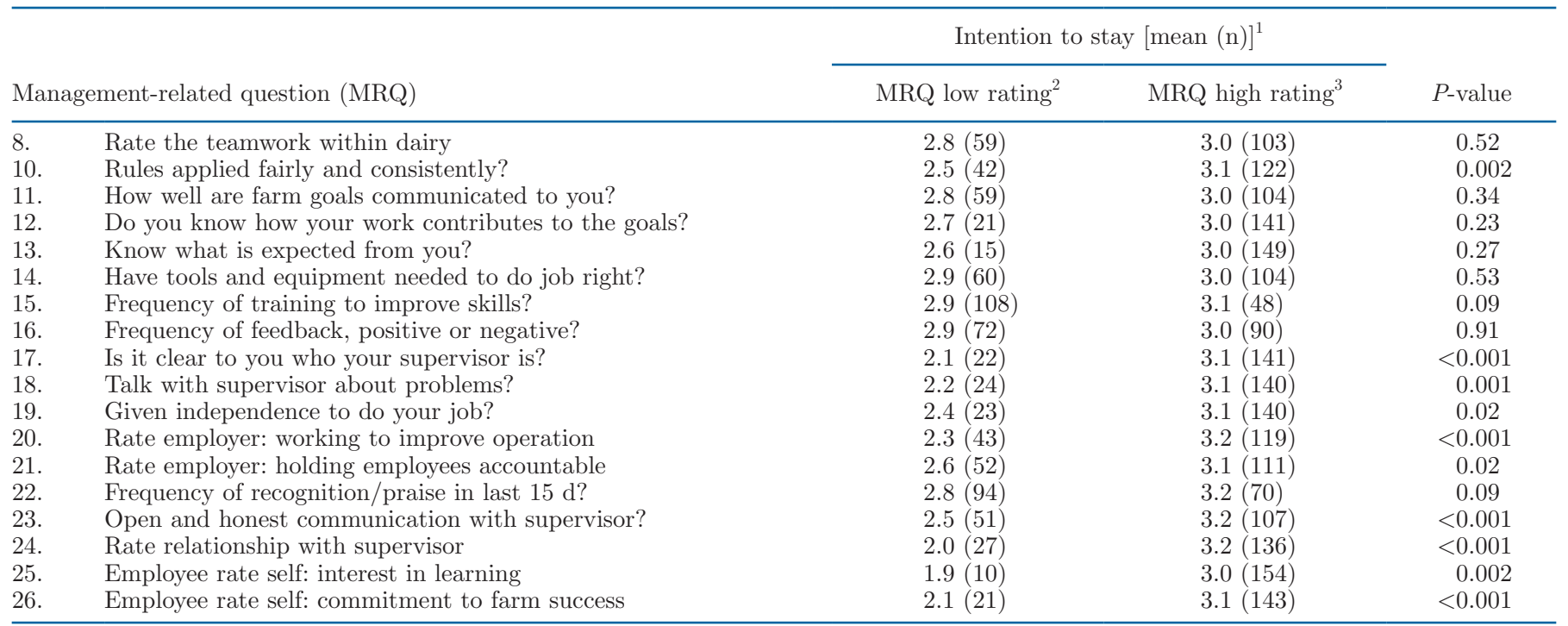

${ }^{1}$ Mean employees' intention to remain at their current place of employment: $1: \leq 1$ yr; $2: 2-3$ yr; $3: 4-5$ yr; and 4: until retirement.

${ }^{2} \mathrm{MRQ}$ low rating: employees rated the MRQ at $1-3$.

${ }^{3} \mathrm{MRQ}$ high rating: employees rated the MRQ at $4-5$.

median response were 3.0 and 3.0 , respectively, on an ordinal scale of 1 to 4 , where $1=$ less than $1 \mathrm{yr}, 2=$ $2-3 \mathrm{yr}, 3=4-5 \mathrm{yr}$, and $4=$ until retirement (Table 2).

Employees who rated their employer higher on MRQs reported a higher score for intention to remain on the job compared with employees who rated their employer lower on the same questions (10 of 18 questions; Table
2 ). When comparing questions where employees answered 1-3 versus $4-5$, employees' intention to remain on the job was most positively correlated with their answers to "rate relationship with supervisor" (Q24; 2.0 vs. 3.2, low MRQ vs. high MRQ, respectively), "employees rate self: interest in learning" (Q25; 1.9 vs. 3.0), "employee rate self: commitment to farm success" (Q26;

Table 3. Employees' willingness to recommend the farm to others as a place to work in relation to their perception of management strengths and weaknesses

\begin{tabular}{|c|c|c|c|}
\hline \multirow{2}{*}{ Management-related question (MRQ) } & \multicolumn{2}{|c|}{ Average recommend ${ }^{1}[$ mean $(\mathrm{n})]$} & \multirow[b]{2}{*}{$P$-value } \\
\hline & MRQ low rating ${ }^{2}$ & MRQ high rating ${ }^{3}$ & \\
\hline Rate the teamwork within dairy & $3.9(61)$ & $4.3(104)$ & $<0.01$ \\
\hline Rules applied fairly and consistently? & $3.5(43)$ & $4.4(125)$ & $<0.001$ \\
\hline How well farm goals communicated to you? & $3.9(60)$ & $4.3(107)$ & 0.01 \\
\hline Do you know how your work contributes to the goals? & $3.5(21)$ & $4.2(145)$ & 0.01 \\
\hline Know what is expected from you? & $3.4(16)$ & $4.2(152)$ & 0.01 \\
\hline Have tools and equipment needed to do job right? & $4.0(60)$ & $4.2(107)$ & 0.13 \\
\hline Frequency of training to improve skills? & $4.1(110)$ & $4.3(50)$ & 0.22 \\
\hline Frequency of feedback, positive or negative? & $4.2(73)$ & $4.1(93)$ & 0.88 \\
\hline Is it clear to you who your supervisor is? & $3.5(23)$ & $4.3(144)$ & 0.001 \\
\hline Talk with supervisor about problems? & $3.2(25)$ & $4.3(143)$ & $<0.001$ \\
\hline Given independence to do your job? & $3.5(24)$ & $4.3(143)$ & $<0.01$ \\
\hline Rate employer: working to improve operation & $3.4(44)$ & $4.4(122)$ & $<0.001$ \\
\hline Rate employer: holding employees accountable & $3.7(53)$ & $4.4(114)$ & $<0.001$ \\
\hline Frequency of recognition/praise in last $15 \mathrm{~d} ?$ & $3.9(96)$ & $4.5(72)$ & $<0.001$ \\
\hline Open and honest communication with supervisor? & $3.6(52)$ & $4.4(109)$ & $<0.001$ \\
\hline Rate relationship with supervisor & $3.2(28)$ & $4.3(139)$ & $<0.001$ \\
\hline Employee rate self: interest in learning & $3.7(10)$ & $4.2(158)$ & 0.22 \\
\hline Employee rate self: commitment to farm success & $3.3(22)$ & $4.3(145)$ & $<0.001$ \\
\hline
\end{tabular}

${ }^{1}$ Mean employees' likelihood to recommend this workplace to others on a scale from 1 to 5 , where $1=\mathrm{I}$ would discourage others from working here; 5 = I would strongly recommend this place to them.

${ }^{2} \mathrm{MRQ}$ low rating: employees rated the MRQ at 1-3.

${ }^{3} \mathrm{MRQ}$ high rating: employees rated the MRQ at $4-5$. 
2.1 vs 3.1), and "is it clear to you who your supervisor is" (Q17; 2.1 vs 3.1; Table 2). There was no association $(P>0.05)$ between employees' intention to stay and their answers to questions about teamwork (Q8), goals (Q11 and Q12), clarity of their job (Q13), tools to do the job (Q14), or frequency of feedback (Q16 and Q22).

In the univariable analyses, employees' intention to stay was positively associated with their duration of employment on the farm. Employees who identified their role as milking intended to remain on the job for a shorter interval (Table 5). English-speaking employees' intention to remain on the farm tended to be higher than that of Spanish-speaking employees (Table 5). Employees' intention to stay was also positively associated with the PCA components Relationships, Feedback and Fairness, Goals and Directions, and Self-Appraisal (Table 6). In the multivariable analysis, length of employment, and the components Relationships and Self-Appraisal were positively correlated with employees' intention to stay on the farm (Table 7).

\section{Willingness to Recommend}

For Q7: Would you recommend this workplace to others? mean and median response were 4.2 and 4.0, respectively, on an ordinal scale of $1-5$ (Table 3 ), where $1=\mathrm{I}$ would discourage others from working here and 5 = I would strongly recommend this place to them.

Employees who rated their employer higher on MRQs reported a higher willingness to recommend the work- place to others compared with employees who rated their employer lower on these same questions (14 out of 18 questions; Table 3). When comparing questions where employees answered 1-3 versus $4-5$, employees' willingness to recommend the workplace to others was most positively correlated with the relationship-related questions "rate relationship with supervisor" (Q24; 3.2 vs. 4.3, low MRQ vs. high MRQ, respectively), "talk with supervisor about problems" (Q18; 3.2 vs. 4.3), and "rate employer: working to improve the operation" (Q20; 3.4 vs. 4.4). Additionally, "employee rate self: commitment to farm success" (Q26) showed a strong positive correlation with employees' willingness to recommend the workplace to others (3.3 vs. 4.3). Willingness to recommend the workplace to others was not positively correlated with having the tools to do the job (Q14), frequency of training (Q15), frequency of feedback (Q16), or an employee's rating of their own interest in learning (Q25; Table 3).

In the univariable analysis, tools/equipment, training, independence to do the job, and components Relationships, Feedback and Fairness, Goals and Directions, and Self-Appraisal were positively correlated with employees' willingness to recommend the farm as a workplace (Tables 5 and 6). The odds of Spanish-speaking employees recommending their place of work to others were almost twice that of their English-speaking coworkers (Table 5). In the multivariable analysis, the components Relationships and Self-Appraisal remained significant (Table 7).

Table 4. Employee engagement in relation to their perception of management strengths and weaknesses

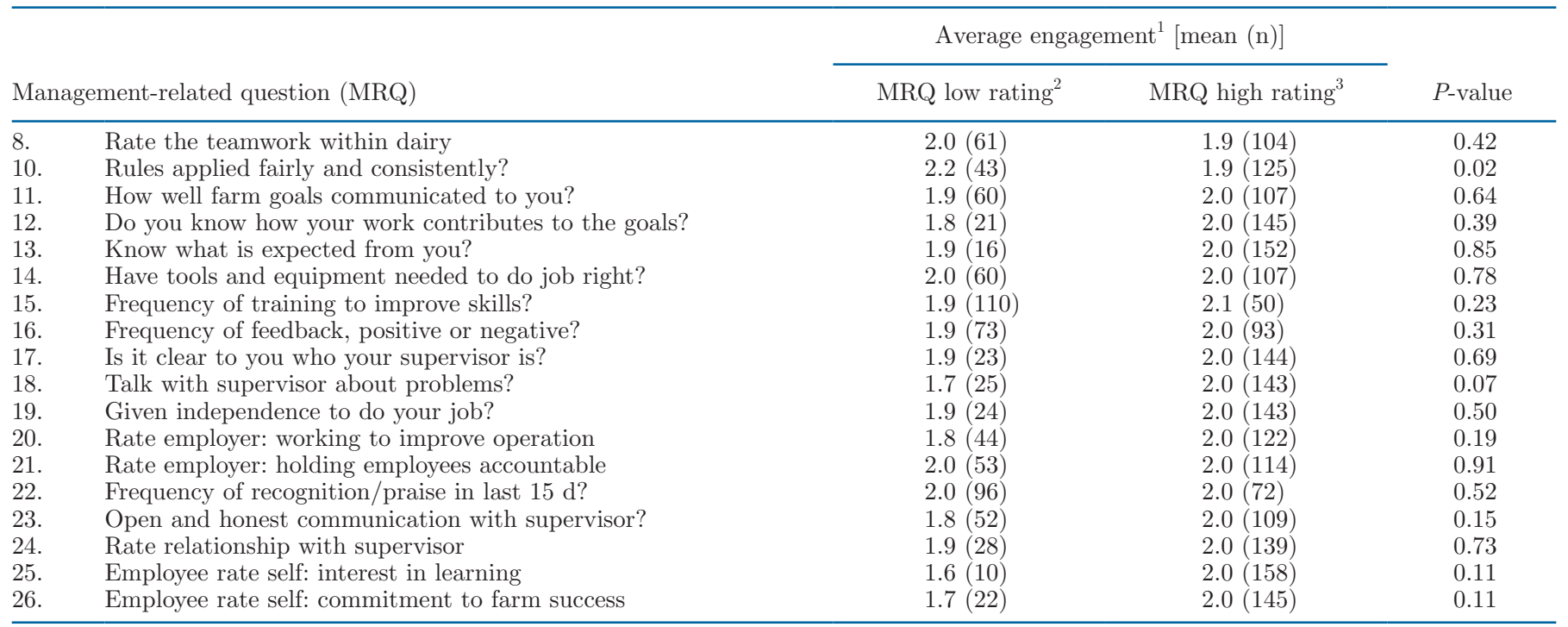

${ }^{1}$ Mean engagement defined as employees having ideas to improve the business and sharing them with management, where $1=$ don't have ideas or have ideas but don't share them; $2=$ have ideas occasionally and share them; 3 = have ideas frequently and share them.

${ }^{2} \mathrm{MRQ}$ low rating: employees rated the MRQ at $1-3$.

${ }^{3} \mathrm{MRQ}$ high rating: employees rated the MRQ at $4-5$. 
Moore et al.: MANAGEMENT'S IMPACT ON EMPLOYEE SATISFACTION

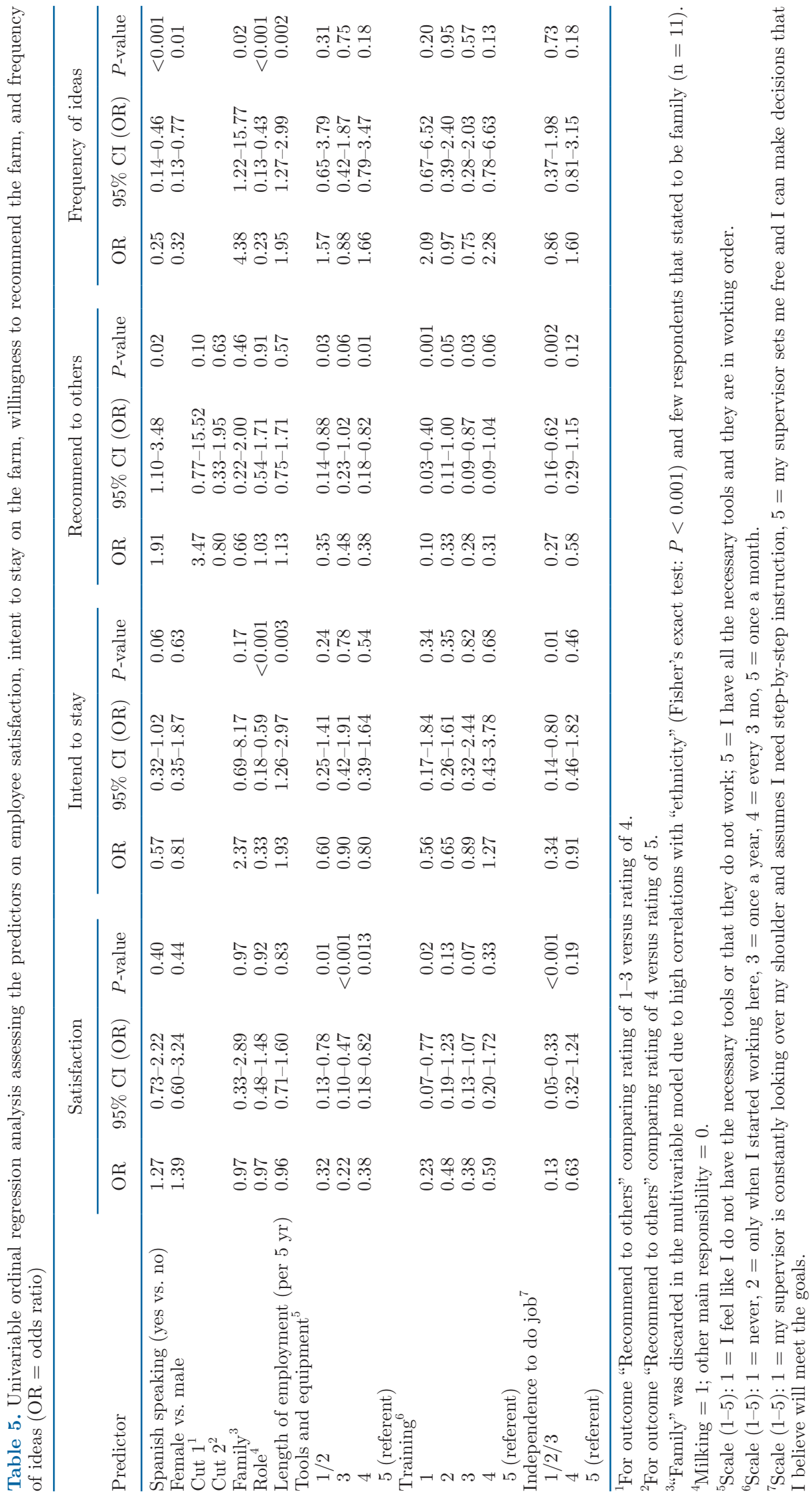



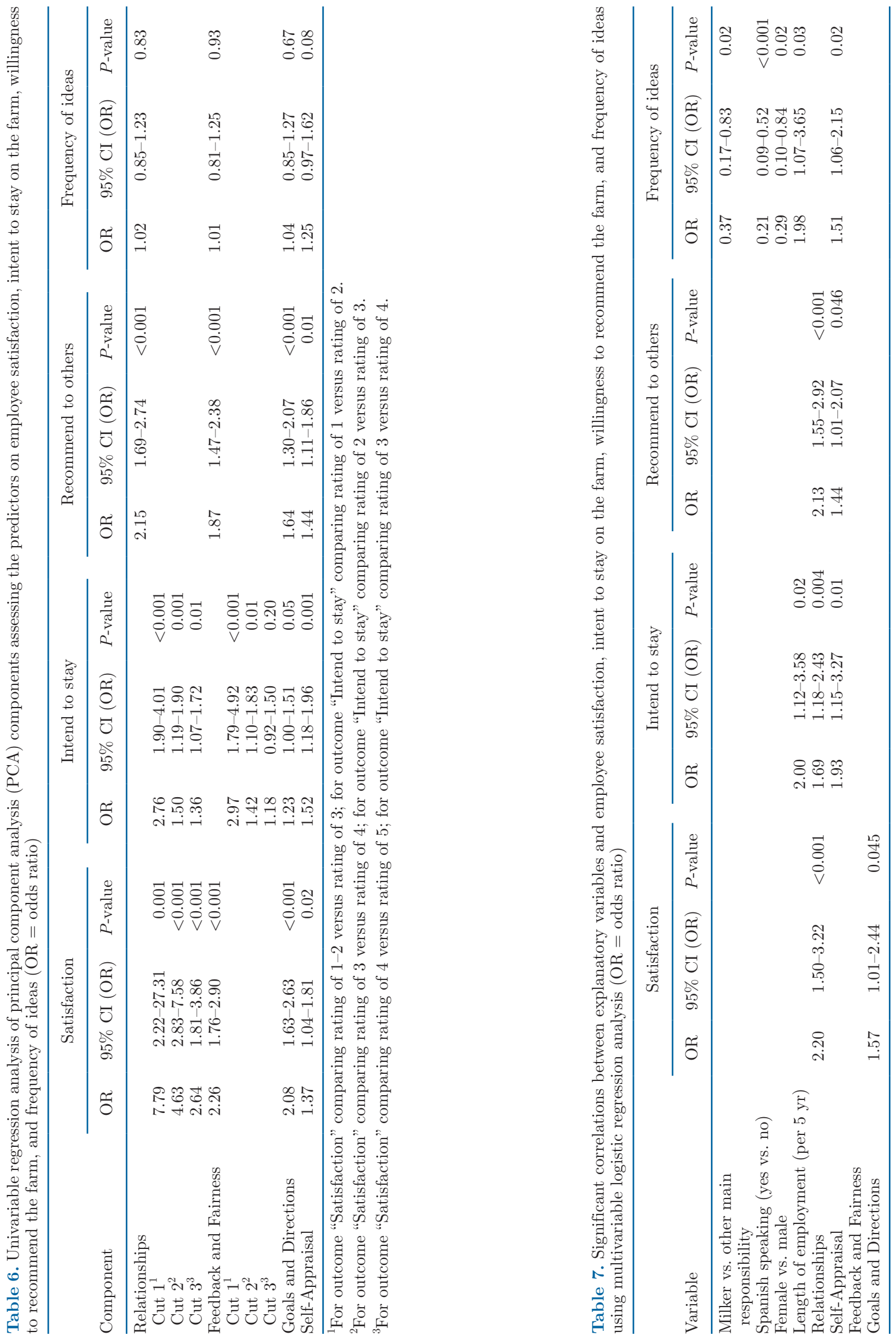


\section{Level of Employee Engagement}

For Q27: How often do you come up with ideas to improve the operation (whether you share these or not)? mean and median response were 2.0 and 2.0 on an ordinal scale of 1-3 (Table 4 ), where $1=$ don't have ideas or have ideas but don't share them, $2=$ have ideas occasionally and share them, and $3=$ have ideas frequently and share them.

The frequency of coming up with ideas was only associated with "rules applied fairly and consistently" (Q10; 2.2 vs. 1.9 , low MRQ vs. high MRQ, respectively), with those rating "rules applied fairly and consistently" higher also rating their frequency of sharing ideas lower.

Univariable analysis (Table 5) demonstrated that the frequency of having and sharing ideas was associated with language and gender (i.e., Spanish-speaking employees and women were less likely to voice ideas) and with position on the farm (i.e., milkers were less likely to voice ideas). Employees who had worked on a farm longer were also more likely to share their ideas.

In multivariable analysis, milkers were less likely to share their ideas, as were women and Spanish-speaking employees. Employees with a longer duration of employment were more likely to share ideas (Table 7 ).

\section{DISCUSSION}

Responses of employees on the 12 large US dairy farms to questions that included both assessments of management areas on the farm and effects of those management practices on employees highlighted several areas that may affect employee satisfaction, intention to stay, and whether or not an employee would recommend the farm to others as a place of employment. Within and across each of these 3 areas, employees' answers to certain MRQs had great impact. Relationship with supervisor (Q24) was most strongly and positively correlated with employee satisfaction, intention to stay, and likelihood to recommend the farm to other potential employees. The combined effect of these 3 areas can have a profound effect on a farm's ability to recruit and retain a reliable and productive workforce.

In our study, the Relationship component was positively related to employees' satisfaction, their intention to remain on the farm, and their recommendation of the farm to others (Table 7). This corresponds well to the basic psychological need for "relatedness" (Fowler, 2014). If farmers want to retain employees, it is important that they pay attention to the working environment (Nettle, 2018).

Employees' rating of their satisfaction was related to their answers to almost all MRQs. Those MRQs about their relationship with their supervisor had the stron- gest relationship with the employees' rating of their job satisfaction. This dynamic can lead to high satisfaction, even on some farms where employees scored low in a few management areas, and to low satisfaction on some farms that lead their peers in other management areas (Durst et al., 2018). The components Relationships and Goals and Directions were significant in the multivariable analysis. Employees who had a good relationship with their employer and who understood goals, directions, and how their work fit into accomplishment of those goals were more likely to be satisfied in their jobs. The Goals and Directions component would certainly affect an employee's feeling of autonomy, and so the relationship to satisfaction certainly makes logical sense. The lack of correlation between "satisfaction" and "frequency of feedback, positive or negative" (Q16) may be attributable to the type of feedback the employees were reporting. Despite getting frequent feedback, that alone does not indicate the effectiveness of it, as evidenced by comments such as "negative feedback - always, positive feedback - never."

It is often stated that "people don't leave companies, they leave managers" (e.g., Harter and Rigoni, 2013); our research would support this proposition. Employees who rated the Relationship component as high were 1.7 times more likely to intend to stay with their current employer. In addition, employees who had worked for the farm longer also planned to stay longer. Farmers often report high turnover in new hires, hoping to eventually find a good fit for the farm. This may be indicative of poor hiring practices, leading to bad hires, or a lack of time to build a relationship between employee and employer (Nettle, 2018).

Although we did not ask for reasons for intending to remain on the job for a shorter interval, job-related and personal issues could be responsible. Employees who rated high in the Self-appraisal component rated themselves as higher in their interest in learning and commitment to the farm, consistent with their interest in remaining on the farm. Although employers cannot directly cause employees to be more dedicated to the farm or more interested in learning, they can certainly create a culture that promotes both (Malanski et al., 2017; Sahu et al., 2018). These, along with training and tools, are important in helping employees feel competent, the third of Fowler's psychological needs (Fowler, 2014). Just as importantly, employers can negatively affect both of these through poor employee management. This latter is clearly indicated by findings on the relationship between employees' intention to stay and their frequency of ideas to improve the business. Employees who had no ideas or never shared them were much more likely to want to leave the farm within the coming year (60\%) compared with those who frequently 
shared ideas (15\%). Many comments from employees who never had ideas or did not share them indicated that their ideas had been ignored or ridiculed in the past or that they believed that the employers did not want to hear their ideas. Employers who want employees to continue to work on their farm need to ensure they are promoting an environment where employees feel that they are heard and that their input is valued (Stone et al., 2009).

In general, employees who rated management higher on the MRQs were also more likely to recommend the workplace to other potential employees. Complicating the answer to their willingness to recommend the farm to others is whether the current employee saw a potential new employee as a good fit. Often employees cited that they would recommend the farm to others "if they are a hard worker," "if they had experience with livestock," or "if they will do the work right." Good employees want other good employees as team members, and they will not recommend the farm to individuals who they feel are not a good fit. Furthermore, some employee comments indicated that their low rating on recommending the place of employment to others was affected by their relationship with supervisors. Employees are not likely to recommend to others what they feel is a bad place to work (Baruk, 2017). This becomes the farm's reputation and can directly affect the pool of candidates for future hires. Finally, employees who were dedicated to the farm and had a desire to continue to learn more (high Self-Appraisal component) were willing to recommend the farm to others who they believed would also be committed to the farm's success.

Frequency of "ideas to improve the business" was used as an indicator of how engaged an employee was on the farm, because it demonstrated whether they bring their minds, as well as their bodies, to work and whether they are seeking the good of the employer. It also provided information on how such engagement might be encouraged or discouraged by management. Frequency of ideas was positively associated with the MRQ "rules applied fairly and consistently" (Q10); it was also associated with several employee characteristics, as well as the Self-Appraisal component. Individuals who were in a milking role, Spanish speaking, or female stated that they provided less feedback to employers in the form of ideas to improve the business (Table 7). Additionally, length of employment was positively related to frequency of ideas, suggesting that employees with more tenure either have more ideas to share, feel more comfortable sharing ideas, or know the farm well enough to be able to identify issues. As milking roles tended to be filled by Spanish-speaking employees with fewer years of experience, it is difficult to separate out the effects of each of these factors. Finally, frequency of ideas was positively associated with the Self-Appraisal component; that is, employees' interest in learning and employees' commitment to the farm. Individuals who were committed to the dairy and interested in learning indicated that they were more likely to have ideas to share and felt compelled to share those ideas to improve the operation. We recognize that "frequency of ideas" is only one measurement of employee engagement. Other factors such as showing up on time, following protocols thoroughly, engaging in training, and so on, all contribute to an engaged employee. However, the comments that lead to the "frequency of ideas" rating did shed light on the effects that management can have to either encourage or quell an employee's engagement.

Despite the association between the 3 outcomes, employee ratings of the various management areas did not have the same impact on each outcome. Satisfaction, intention to stay, and willingness to recommend the farm to others were complicated by measures beyond the scope of this research; even within the scope of our research, farm-specific levels of failure or success in individual management areas do not stand on their own. For example, one farm may be exceptional at fairly and consistently applying the rules but fail miserably at allowing employees the independence to do their jobs (Durst et al., 2018). This farm's employees may report a lower satisfaction than a farm that does a modestly good job in both of these areas. Thus, farms cannot rely on one trait that they are inherently good at but should instead seek to improve in all areas.

A limitation to the interpretation of our results was that the 12 farms that participated in the study constituted a convenience sample, where farm owners agreed to let employees volunteer to participate. The nature of this enrollment could have introduced bias in the selection of farms as being representative of the US dairy industry. These farmers were interested in improved employee management, which may not be indicative of all farmers. Employee responses were based on each employee's perception of management on the day they were interviewed. Employee perceptions may not be indicative of actual management practices.

\section{CONCLUSIONS}

Our research provided further insight into the influence of employee perceptions of HRM strengths and weakness and their influence on employees' satisfaction in their work, desire to continue to work on the farm, and whether they would recommend employment on the farm to others. The importance of an employee's relationship with their supervisor and other employees cannot be overstated. These relationships can have a positive or negative effect on satisfaction, longevity, 
and recruitment. Although we feel that employees' willingness to share ideas with management is a measure of their engagement, we also recognize that this measurement is affected by other factors, including culture, gender, role on the farm, and duration of employment. Other critical measurements of engagement should be characterized to give insight in how employers can build a culture that encourages engagement. Farms that nurture workers to have a high interest in learning and high commitment to the farm's success can expect workers to provide feedback that will help the farm meet its goals. Results of this study can be used by farm owners in improving recruiting processes, emphasizing strengths of that particular farm's culture. Other responses can help managers and owners improve their management and thereby retain good employees. Future studies based on more employee input should further examine factors that affect employee engagement.

\section{ACKNOWLEDGMENTS}

This study was supported by the North Central Risk Management Education Center and USDA National Institute of Food and Agriculture (Washington, DC) under award number 2010-49200-06200. Felix Soriano, chief executive officer of APN Consulting (Easton, PA), was a partner in enrolling and working with herds outside of Michigan. We greatly appreciate the support of all participating dairy employees, farmers, and managers. The authors have not stated any conflicts of interest.

\section{REFERENCES}

Adcock, F., D. Anderson, and P. Rosson. 2015. The economic impacts of immigrant labor on U.S. dairy farms. Center for North American Studies, Texas A\&M University. Accessed Nov. 24, 2019. http: //www.nmpf.org/files/immigration-survey-090915.pdf.

Babbie, E. R. 2015. The Practice of Social Research. 14th ed. Wadsworth Publishing, Boston, MA.

Barkema, H. W., M. A. G. von Keyserlingk, J. P. Kastelic, T. J. G. M. Lam, C. Luby, J.-P. Roy, S. J. LeBlanc, G. P. Keefe, and D. F. Kelton. 2015. Invited review: Changes in the dairy industry affecting dairy cattle health and welfare. J. Dairy Sci. 98:7426-7445. https://doi.org/10.3168/jds.2015-9377.

Baruk, A. 2017. Contentment of employees vs their prosumeric activity in the scope of recommending an employer. J. Bus. Ind. Mark. 32:742-751. https://doi.org/10.1108/JBIM-10-2016-0240.

Brown, T. A. 2014. Confirmatory Factor Analysis for Applied Research. 2nd ed. Guilford Publications, New York, NY.

Durst, P. T., S. J. Moore, C. Ritter, and H. W. Barkema. 2018. Evaluation by employees of employee management on large US dairy farms. J. Dairy Sci. 101:7450-7462. https://doi.org/10.3168/jds .2018-14592.

Erskine, R. J., R. O. Martinez, and G. A. Contreras. 2015. Cultural lag: A new challenge for mastitis control on dairy farms in the United States. J. Dairy Sci. 98:8240-8244. https://doi.org/10 $.3168 /$ jds.2015-9386.
Fabrigar, L. R., and D. T. Wegener. 2011. Exploratory Factor Analysis. Oxford University Press, New York, NY.

Fowler, S. 2014. Why motivating people doesn't work ... and what does. Berrett-Koehler Publishers Inc., San Francisco, CA.

Harter, J., and B. Rigoni. 2013. State of the American manager, analytics and advice for leaders. Accessed Nov. 24, 2019. https://www .gallup.com/workplace/238085/state-american-workplace-report -2017 .aspx.

Hoyle, R. 2012. Confirmatory factor analysis. Pages 465-497 in Handbook of Applied Multivariate Statistics and Mathematical Modeling. Accessed Nov. 24, 2019. https://doi.org/10.1016/B978 -012691360-6/50017-3.

Ilardi, B. C., D. Leone, T. Kasser, and R. M. Ryan. 1993. Employee and supervisor ratings of motivation: Main effects and discrepancies associated with job satisfaction and adjustment in a factory setting. J. Appl. Soc. Psychol. 23:1789-1805. https://doi.org/10 $.1111 /$ j.1559-1816.1993.tb01066.x.

Malanski, P. D., N. Hostiou, and S. Ingrand. 2017. Evolution pathways of employees' work on dairy farms according to task content, specialization, and autonomy. Cah. Agric. 26:65005. https://doi.org/ $10.1051 /$ cagri/2017052.

Nettle, R. 2018. International trends in farm labour demand and availability (and what it means for farmers, advisers, industry and government). Pages 76-80 in Proc. Int. Agric. Workforce Conf., Cork, Ireland. Moorepark Animal and Grassland Research and Innovation Centre, Teagasc, Moorepark, Fermoy, Co. Cork, Ireland.

Ritter, C., J. Jansen, S. Roche, D. F. Kelton, C. L. Adams, K. Orsel, R. J. Erskine, G. Benedictus, T. J. G. M. Lam, and H. W. Barkema. 2017. Invited review: Determinants of farmers' adoption of management-based strategies for infectious disease prevention and control. J. Dairy Sci. 100:3329-3347. https://doi.org/10.3168/ jds.2016-11977.

Ryan, R. M., and E. L. Deci. 2000. Self-determination theory and the facilitation of intrinsic motivation, social development, and wellbeing. Am. Psychol. 55:68-78. https://doi.org/10.1037/0003-066X .55 .1 .68 .

Sahu, S., A. Pathardikar, and A. Kumar. 2018. Transformational leadership and turnover: Mediating effects of employee engagement, employer branding, and psychological attachment. Leadersh. Organ. Dev. J. 39:82-99. https://doi.org/10.1108/LODJ-12-2014 $-0243$.

Stone, D., E. L. Deci, and R. M. Ryan. 2009. Beyond talk: Creating autonomous motivation through self- determination theory. J. Gen. Manage. 34:75-91. https://doi.org/10.1177/030630700903400305.

Stup, R. E., J. Hyde, and L. A. Holden. 2006. Relationships between selected human resource management practices and dairy farm performance. J. Dairy Sci. 89:1116-1120. https://doi.org/10.3168/ jds.S0022-0302(06)72180-4.

Tavakol, M., and R. Dennick. 2011. Making sense of Cronbach's alpha. Int. J. Med. Educ. 2:53-55. https://doi.org/10.5116/ijme.4dfb $.8 \mathrm{dfd}$.

USDA-NAHMS. 2016. Dairy 2014: Dairy cattle management practices in the United States, 2014. Accessed Nov. 24, 2019. https://www .aphis.usda.gov/animal_health/nahms/dairy/downloads/dairy14/ Dairy14_dr_PartI.pdf.

Van den Broeck, A., M. Vansteenkiste, H. De Witte, B. Soenens, and W. Lens. 2010. Capturing autonomy, competence, and relatedness at work: Construction and initial validation of the Work-related Basic Need Satisfaction scale. J. Occup. Organ. Psychol. 83:9811002. https://doi.org/10.1348/096317909X481382.

\section{ORCIDS}

Stanley J. Moore (ำ https://orcid.org/0000-0003-2817-8575 Phillip T. Durst (1) https://orcid.org/0000-0002-7028-8279 Caroline Ritter @ https://orcid.org/0000-0001-7349-5241 Diego Nobrega ๑ https://orcid.org/0000-0001-9821-1436 Herman W. Barkema @ https://orcid.org/0000-0002-9678-8378 